Jerry da Ressureição Geraldo Inácio

Simulação do Regime Intermitente em Tubulações Verticais Utilizando o Modelo de Dois Fluidos com Diferentes Relações de Fechamento

Dissertação de Mestrado

Dissertação apresentada ao Programa de PósGraduação em Engenharia Mecânica da PUC-Rio como requisito parcial para obtenção do título de Mestre em Engenharia Mecânica.

Orientador: Prof. Angela Ourivio Nieckele Co-orientador: João Neuenschwander Escosteguy Carneiro

Rio de Janeiro 


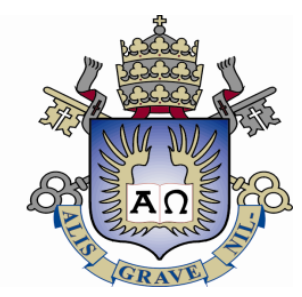

Jerry da Ressureição Geraldo Inácio

\section{Simulação do Regime Intermitente em Tubulações Verticais Utilizando o Modelo de Dois Fluidos com Diferentes Relações de Fechamento}

Dissertação apresentada como requisito parcial para obtenção do grau de Mestre pelo Programa de Pósgraduação em Engenharia Mecânica do Departamento de Engenharia Mecânica do Centro Técnico Cientifico da PUC-Rio. Aprovada pela Comissão Examinadora abaixo assinada.

Profa. Angela Ourivio Nieckele

Orientadora

Departamento de Engenharia Mecânica -PUC- Rio

Dr. João Neuenschwander Escoteguy Carneiro Co-Orientador Sintef do BRASIL

Prof. Eugênio Spano Rosa UNICAMP

Dr. Igor Braga Departamento de Engenharia Mecânica -PUC- Rio

Prof. José Eugenio Leal Coordenador Setorial do Centro

Técnico Científico - PUC-Rio

Rio de Janeiro, 13 de Setembro de 2012 
Todos os direitos reservados. É proibida a reprodução total ou parcial do trabalho sem autorização da universidade, do autor e do orientador.

\section{Jerry da Ressureição Geraldo Inácio}

Graduou-se em Engenharia de Petróleo no Depto. de Engenharia de Petróleo da UGF (Universidade Gama Filho do Rio de Janeiro), em 2010. Atualmente trabalhando no Programa de simulação numérica de escoamento Bifásico em tubulação vertical.

Ficha Catalográfica

Inácio, Jerry da Ressureição Geraldo.

Simulação do regime intermitente em tubulações verticais utilizando o modelo de dois fluidos com diferentes relações de fechamento / Jerry da Ressurreição Geraldo Inácio; orientador: Angela Ourivio Nieckele ; co-orientador: João Neuenschwander Escosteguy Carneiro. - 2012.

110 f. ; $30 \mathrm{~cm}$

Dissertação (mestrado)-Pontifícia Universidade Católica do Rio de Janeiro, Departamento de Engenharia Mecânica, 2012.

Inclui bibliografia

1. Engenharia mecânica - Teses. 2. Escoamento bifásico. 3. Escoamento intermitente. 4. Tubulação vertical. 5. Unidimensional. 6. Modelo de dois fluidos. 7. Relações de fechamento. I. Nieckele, Angela Ourivio. II. Carneiro, João Neuenschwander Escosteguy. III. Pontifícia Universidade Católica do Rio de Janeiro. Departamento de Engenharia Mecânica. IV. Título. 


\section{Agradecimentos}

A minha mãe e aos meus irmãos por seu amor, credibilidade e apoio incondicional.

A Edna Patricia Soares da Costa, minha noiva por todo o seu apoio nos momentos cruciais deste trabalho.

A Angela Ourivio Nieckele, minha orientadora, pela enorme paciência, amizade, conhecimento prestado em fim foi muito bom trabalhar com você durante estes anos.

Ao meu co-orientador João Neuenschwander Escoteguy Carneiro, Pelos conhecimentos prestados, pelo tempo dedicado a este trabalho, paciência e amizade.

Ao João Henrique Paulino de Azevedo pela ajuda na realização dos pósprocessamentos dos dados.

Agradecimentos em particular a PUC-Rio e aos órgãos de fomento à pesquisa CNPq, e CAPES. 


\section{Resumo}

Inácio, Jerry R. G; Nieckele, Angela, O; Carneiro, João N.E. Simulação do Regime Intermitente em Tubulações Verticais Utilizando o Modelo de Dois Fluidos com Diferentes Relações de Fechamento. Rio de Janeiro, 2012. 110p. Dissertação de Mestrado - Departamento de Engenharia Mecânica, Pontifícia Universidade Católica do Rio de Janeiro.

Escoamentos intermitentes bifásicos são caracterizados pela sucessão de golfadas de líquido separadas por bolhas de diferentes tamanhos, escoando com frequência variável. A previsão deste tipo de escoamento é muito importante, uma vez que o mesmo pode ser encontrado em diversas aplicações industriais. No presente trabalho, é realizado um estudo do regime intermitente em tubulações verticais, utilizando o Modelo de Dois Fluidos uni-dimensional, juntamente como o método de Volumes Finitos. O Modelo de Dois Fluidos unidimensional requer a inclusão de diversas relações de fechamento para as fases líquida e gasosa. Estas relações devem representar realisticamente o fenômeno físico de interesse, e as equações resultantes devem ser bem postas. No presente trabalho, duas relações de fechamento foram consideradas: salto de pressão devido à curvatura da interface e parâmetro de distribuição de fluxo de quantidade de movimento. A presença do salto de pressão não se mostrou significativa, enquanto que o parâmetro relacionado a distribuição da fase líquida ampliou a região em que o sistema de equações é bem posto. Para os casos analisados, o parâmetro de distribuição da fase líquida apresentou influência nas características do escoamento. As principais grandezas do regime estatisticamente permanente, como comprimento e velocidade de translação da golfada e da bolha, foram comparadas com dados experimentais disponíveis na literatura, apresentando boa concordância.

\section{Palavras-chave}

Escoamento Bifásico; Escoamento Intermitente; Tubulação Vertical; Unidimensional; Modelo de Dois Fluidos; Relações de Fechamento. 


\section{Abstract}

Inácio, Jerry R. G; Nieckele, Angela, O; Carneiro, João N.E (Advisor). Simulation of Intermittent Flow in Vertical Pipes using the Two-Fluid Model with Different Closure Parameters. Rio de Janeiro, 2012. 110p. MSc. Dissertation - Departamento de Engenharia Mecânica, Pontifícia Universidade Católica do Rio de Janeiro.

Intermittent two phase flows are characterized by a succession of liquid slugs separated by bubbles of different sizes, with varying frequency. The prediction of this type of flow is very important, since it can be found in several industrial applications. In the present work, studies of intermittent flows in vertical pipes are performed, employing the Two Fluid Model along with the Finite Volume method. The one-dimensional Two Fluid Model requires the inclusion of several closure relations for both gas and liquid phases. These relations should realistically represent the physical phenomenon of interest, and the resulting equations should be well posed. In this work, two closure relations were considered: pressure jump due to the interface curvature and momentum flux distribution parameter. The inclusion of the pressure jump did not present a significant influence, whereas the momentum flux parameter of the liquid phase increased the region in which the system of equations is well posed. For the cases analyzed, the momentum flux parameter of the liquid phase affected the flow characteristics. The main statistical slug quantities, such as length and translational speed of the bubble and slug, were compared with experimental data available in the literature, showing good agreement.

\section{Keywords}

Two-Phase Flow; Slug Flow; Vertical Pipe; One-Dimensional; Two Fluid Model; Closure Parameters. 


\section{Sumário}

$\begin{array}{lr}\text { 1. Introdução } & 17\end{array}$

$\begin{array}{lr}\text { 1.1. Regime Intermitente } & 19\end{array}$

1.2. Grandezas Caracteristicas das Golfadas 21

1.3. Objetivo 22

1.4. Organização do Trabalho 22

2. Revisão Bibliográfica $\quad 24$

2.1. Escoamento Intermitente 24

2.2.Simulação Numérica do Escoamento Intermitente 28

3. Modelo Matemático 33

3.1.Equações de Conservação 35

3.2 Parâmetros Geométricos 36

3.2.1.Escoamento Estratificado 36

3.2.2. Escoamento Anular 37

3.3. Equações de Estado 38

3.4. Tensões Cisalhantes 38

3.5. Parâmetro de Fluxo de Quantidade de Movimento $\left(\boldsymbol{C}_{\boldsymbol{k}}\right) \quad 40$

3.6 Distribuição da Pressão Hidrostática 40

3.7.Salto de Pressão na Interface 42

3.7.1. Escoamento Estratificado 42

3.7.2. Escoamento Anular 43

3.8. Equações de Conservação de Quantidade de Movimento Linerar 44

3.9. Análise de Hiperbolicidade 45

4. Método Numérico 53

4.1. Fração Volumétrica $\quad 54$

4.2. Velocidades $\quad 55$

4.3. Pressão 58

4.4. Volumes de Controle das Fronteiras 59

4.5. Malha Computacional e Criterios para o Passo de Tempo 60

4.6. Critério de Convergência 61

4.7. Procedimento de Execução 62

4.8. Formação da Golfada 63 
4.9. Cálculos dos Parâmetros Médios das Golfadas 64

5. Resultados 67

5.1 Tubulação Horizontal $\quad 67$

$\begin{array}{ll}5.2 \text { Tubulação Vertical } & 71\end{array}$

5.2.1. Análise do bom/mal condicionamento para escoamentos verticais 73

5.2.2. Influência dos parâmetros de fechamento 77

5.3. Configuração 1

5.4. Configuração $2 \quad 98$

6. Comentarios Finais 100

6.1. Conclusões 101

6.2. Sugestões para Trabalhos Futuros 102

Referência Bibliográfica 103

$\begin{array}{ll}\text { Apendice A1 } & 110\end{array}$ 


\section{Lista de tabelas}

Tabela 3.1 - Correlações para o fator de atrito. 39

Tabela 5.1- Propriedades do Fluido (Caso H). 67

Tabela 5.2 - Condições de Contorno para Casos Verticais bifásico 72 


\section{Lista de figuras}

Figura 1.1. - Campo de Dália, Angola

Figura 1.2. - Padrões de Escoamento para Fluxos Horizontal e

Vertical

Figura 1.3. - Padrão Intermitente 20

Figura 1.4. - Unidade Básica de uma Golfada 21

Figura 3.1 - Geométria Escoamento Estratificado 37

Figura 3.2. - Geometria do Escoamento Vertical 37

Figura 3.3. - Aproximação Hidrostática para o Perfil de Pressão no Escoamento Estratificado.

Figura 3.4. - Interface Gás-Liquido e Definições Geométricas para o Cálculo da Curvatura Carneiro (2006) 42

Figura 3.5. - Raios de Curvatura da Geometria Anular 44

Figura 4.1 - Volumes de Controle (a) Escalar e (b) Vetorial. 53

Figura 4.2. - Volumes de Controle (a) Entrada e (b) Saída 60

Figura 4.3. - Fluxograma Esquemático da Execução 63

Figura 4.4. - llustrações das Medições das Velocidades e Comprimento. 65

Figura 5.1. - Limite entre bem/mal posto para valores diferente do parâmetro de fluxo de quantidade de movimento $\left(C_{L}\right.$ e $\left.C_{G}\right)$, para escoamento horizontal.

Figura 5.2. - - Influência do parâmetro de fluxo de quantidade de movimento $C_{L}$, em função do espaçamento da malha. (a) holdup de líquido. (b) frequência de translação da golfada.

Figura 5.3. - Influência do parâmetro de fluxo de quantidade de movimento $C_{L}$, em função do espaçamento da malha.(a) comprimento da unidade de golfada (b) velocidade de translação da golfada $\quad 71$ Figura 5.4. - Esquema dos experimentos. 72

Figura 5.5. - Mapa de padrões de escoamento para as duas configurações e os casos analisados.

Figura 5.6. - Influência do valor de $C_{L}$ na linha de bem posto para escoamento vertical . 
Figura 5.7. - Influência do valor de $C_{G}$ nos limites de bom $/ \mathrm{mal}$ condicionamento do modelo para escoamento vertical I

Figura 5.8. - Influência da curvatura transversal no salto de pressão nos limites de bom/mal condicionamento do modelo para escoamento vertical.

Figura 5.9. - Influência do comprimento de onda nos limites de bom/mal condicionamento do modelo para escoamento vertical.

Figura 5.10. - Influência dos termos de fechamento, juntamente com teste de malha para o duto curto.

Figura 5.11. - Influência dos termos de fechamento, juntamente com teste de malha para o duto real.

Figura 5.12. - Influência dos termos de fechamento na evolução espacial para Caso \#1.

Figura 5.13. - Evolução temporal do holdup médio de líquido e o desvio padrão de para vários valores dos parâmetros de fluxo de quantidade de movimento do líquido..

Figura 5.14. - Influência do parâmetro de distribuição de fluxo de quantidade de movimento de líquido na evolução temporal do holdup de líquido instantâneo.

Figura 5.15. - Influência do parâmetro de distribuição de fluxo de quantidade de movimento de líquido na evolução espacial para Caso \#1.

Figura 5.16. - Influência do parâmetro de distribuição de fluxo de quantidade de movimento de líquido próximo à saída do duto para Caso \#1.

Figura 5.17. - Distribuição de pressão ao longo da seção de teste para o Caso \# 1 .

Figura 5.18. - Evolução espacial para o Caso \#1. 87

Figura 5.19. - Histograma e PDFs para o Caso \#1. 89

Figura 5.20. - Comparação de PDFs de comprimento de pistão para Caso \#1

Figura 5.21. - Distribuição ao longo do Duto de algumas variaveis Caso \#1 
Figura 5.22. - Distribuição de pressão ao longo da seção de teste para o Caso \#2 91

Figura 5.23. - Evolução espacial para o Caso \#2. 92

Figura 5.24. - PDFs do comprimento da golfada para o Caso \#2, em $\mathrm{x}=4,68 \mathrm{~m}$.

Figura 5.25. - Distribuição de pressão ao longo da seção de teste para o Caso \#3 e Caso \# 4.

Figura 5.26. - Evolução espacial para o Caso \#3 e Caso \#4.

Figura 5.27. - PDFs do comprimento da golfada e da bolha para o Caso \#3, em $x=4,68 \mathrm{~m}$.

Figura 5.28. - PDFs do comprimento da golfada e da bolha para o Caso \#4, em $\mathrm{x}=4,68 \mathrm{~m}$.

Figura 5.29. - Evolução espacial para o Caso \#5. 98

Figura 5.30. - Evolução espacial para o Caso \#6 99 


\section{Lista de símbolos}

A Área da seção transversal da tubulação

D Diâmetro da tubulação

C Número de Courant

$C_{0} \quad$ Parâmetro de distribuição

$C_{k} \quad$ Parâmetro de fluxo de quantidade de movimento

$f \quad$ Fator de fricção

Fr Número de Froude

$g \quad$ Aceleração da gravidade

$h_{L} \quad$ Altura da superfície do líquido

$L \quad$ Comprimento

$P \quad$ Pressão interfacial e da fase gasosa

$R \quad$ Constante do gás

Re Número de Reynolds

$S \quad$ Perímetro molhado

$t \quad$ Tempo

$T \quad$ Temperatura

$U \quad$ Velocidade

$\dot{\forall} \quad$ Vazão volumétrica

$x \quad$ Coordenada axial

\section{Símbolos gregos}

$\alpha \quad$ Fração volumétrica da fase

$\beta \quad$ Ângulo de inclinação da tubulação com respeito à horizontal

$\Delta \quad$ Variação de uma grandeza

$\gamma \quad$ Fator de subrelaxação

$\mu \quad$ Viscosidade dinâmica 


$\begin{array}{ll}v & \text { Viscosidade cinemática } \\ \sigma & \text { Tensão cisalhante } \\ \pi & \text { Constante } \mathrm{Pi} \\ \rho & \text { Massa específica } \\ \tau & \text { Tensão de cisalhamento }\end{array}$

\section{Subscritos}

e,w Faces leste e oeste do volume de controle principal

E Referente ao centro do volume principal de controle a leste entrada Entrada da tubulação

$G \quad$ Fase gasosa

I lésimo ponto nodal

$i \quad$ Interface

$k \quad$ Relativo a fase $k$

$L \quad$ Fase líquida

$m \quad$ Mistura

max Máximo valor

$P \quad$ Centro do volume de controle principal

ref Referência

$s \quad$ Referente a "slug", ou golfada

saída Saída da tubulação

sk Superficial, relativa a fase $\mathrm{k}$

TP Bifásico

u Unitário

w Parede da tubulação

W Corresponde ao volume de controle oeste ao volume principal

\section{Sobrescritos}

o Referente ao passo de tempo anterior 
ref Referência

$1 \quad$ Grandeza aproximada mediante esquema upwind

Grandeza aproximada mediante interpolação linear

* $\quad$ Referente a iteração anterior 